\title{
PREVALENCIA DEL JUEGO DE APUESTAS EN ADOLESCENTES. UN ANÁLISIS DE LOS FACTORES ASOCIADOS
}

\section{PREVALENCE OF GAMBLING IN ADOLESCENTS. AN ANALYSIS OF THE ASSOCIATED FACTORS}

Pablo Caselles Cámara, Victor Cabrera Perona y Daniel Lloret Irles

Universidad Miguel Hernández, España

\section{Abstract}

Despite the fact that gambling is an activity that is not allowed to minors, international studies found $16 \%$ of adolescents have gambled in the last 12 months, and between $2.5 \%$ and $5.6 \%$ meet criteria for risky gambling. The objective of this paper is to describe the gambling behavior in a sample of adolescents and to analyze the relationship with accessibility and the subjective norm. The gambling behavior, the perception of accessibility and the subjective norm are analyzed in 2,716 students of Secondary Education and Bachelor. The mean age was 15.12 years $(S D=1.03)$. The boys showed higher frequencies in all types of games. Adolescents with the highest frequency of gambling cast significantly higher scores in the accessibility and subjective norm scales ( $p \leq$ .001). We discuss the transfer of the results to the design of preventive interventions. It is suggested that universal prevention should be aimed preferably at children under 15 years old. Attention is drawn to regulators and public administrators on the directly proportional relationship between accessibility and the frequency of gambling.

Keywords: Gambling, adolescent, prevalence, availability, subjective norm

\section{Resumen}

A pesar de que el juego de apuestas es una actividad no permitida a los menores, estudios internacionales calculan que el $16 \%$ de los adolescentes ha jugado en los últimos 12 meses, y entre $2.5 \%$ y $5.6 \%$ cumplen criterios de juego de riesgo. El objetivo del presente trabajo es describir la conducta de juego de apuestas en una muestra de adolescentes y analizar su relación con la accesibilidad y la norma subjetiva. Se analiza la conducta de juego, la percepción de accesibilidad y la norma subjetiva en 2.716 estudiantes de Educación Secundaria Obligatoria y Bachiller. La media de edad fue de 15.12 años (DT $=1.03$ ). Los chicos presentaron frecuencias superiores en todos los tipos de juego. Los adolescentes con mayor frecuencia de juego registran puntuaciones significativamente mayores en las escalas de accesibilidad y norma subjetiva ( $p \leq .001)$. Se discute la aplicación de los resultados en el diseño de las intervenciones preventivas. Se sugiere que la prevención universal debe dirigirse preferentemente a menores de 15 años y se llama la atención a reguladores y administraciones públicas sobre la relación directamente proporcional entre la accesibilidad y la frecuencia de juego.

Palabras clave: Juego de apuestas, adolescentes, prevalencia, accesibilidad, norma subjetiva. 
El juego de apuestas es una actividad en continuo crecimiento. Desde 2011, año en que se regula el juego online, Ley 13/2011 de regulación del juego (Ministerio de Hacienda y Administraciones Públicas, 2011) las apuestas por Internet se han incrementado, compensando la reducción del juego presencial. En los últimos cuatro años, 2013-2017, la facturación del juego online ha crecido a un ritmo medio del 25\% anual (Dirección General de Ordenación de Juego, 2017).

Una amplia mayoría de la población adulta española (75.7\%) ha apostado en los últimos doce meses. De ellos, el $3.5 \%$ muestra conductas de juego de riesgo o problemáticas: $2.6 \%$ son jugadores en riesgo, .6\% jugadores problemáticos y $.3 \%$ jugadores patológicos (Dirección General de Ordenación del Juego, 2015b). Estudios realizados en otros países hallan proporciones similares de población adulta afectada por el juego (Cox, Enns y Michaud, 2004; Gill, Grande y Taylor, 2006; Kessler et al., 2008; Wiebe y Cox, 2005).

A pesar de que el juego de apuestas es una actividad no permitida a los menores, una importante proporción declara haber jugado. Según la encuesta ESPAD que incluye jóvenes de 15 años de 37 países europeos, el 16\% de los adolescentes españoles declara haber jugado en los últimos 12 meses, dos puntos por encima la media europea, y el 7\% haberlo hecho con frecuencia (ESPAD Group, 2016). En lo que se refiere al juego problemático entre menores, las investigaciones encuentran prevalencias que varían entre el .2 y el $12.3 \%$, mostrando valores de un 2.5\%-5.6\% de adolescentes que cumplen criterios de juego de riesgo (Becoña, Míguez y Vázquez, 2001; Calado, Alexandre y Griffiths, 2017; Chóliz y Lamas, 2017; González-Roz, Fernández-Hermida, Weidberg, Martínez-Loredo y Secades-Villa, 2016; Míguez y Becoña, 2015). La heterogeneidad de las muestras, la distinta operativización y el uso de diferentes escalas de medida podrían explicar gran parte de esta variabilidad.

Especialmente preocupante resulta el grado de fidelización entre adolescentes una vez iniciadas las primeras apuestas. Una evaluación longitudinal muestra que la tasa de continuidad de juego tras un año supera el 70\% (Lloret et al., 2018) y la proporción de apostadores que posteriormente desarrollan problemas con el juego es mayor entre aquellos que se inician siendo menores de edad. Así, la proporción de inicio antes de los 18 años aumenta del $13.4 \%$ en jugadores adultos no problemáticos al $44.8 \%$ en jugadores patológicos (Dir. Gral. de Ordenación del Juego, 2015b). Estas cifras son un claro indicador de la importancia de retrasar la edad de inicio para la prevención del juego problemático.

El inicio y mantenimiento del juego de apuestas ha sido relacionado con diferentes variables psicológicas como la impulsividad (Dussault, Brendgen, Vitaro, Wanner y Tremblay, 2011; Vitaro, Arseneault y Tremblay, 1999), la búsqueda de sensaciones (Donati, Chiesi y Primi, 2013), la dificultad para gestionar las propias emociones o baja inteligencia emocional (Potenza et al., 2011), o los sesgos cognitivos en el manejo de las probabilidades (Donati, Chiesi y Primi, 2013). Desde la perspectiva psicosocial, la influencia del entorno social ha sido objeto de investigación en un gran número de conductas potencialmente adictivas y existe evidencia que relaciona la frecuencia de juego de los menores con una mayor conducta parental de apuesta y actitud favorable hacia el juego (Dixon et al., 2016), con una defectuosa supervisión parental (Molinaro et al., 2014), y la percepción de una actitud o conducta a favor del juego de apuestas de parte de los amigos y compañeros cercanos (Lang et al., 2004).

La influencia del entorno social íntimo en la conducta de un individuo ha sido definida como Norma Subjetiva y hace referencia a la presión social percibida para realizar o no un determinado comportamiento (Ajzen, 1987). Este componente es el resultado de la multiplicación de dos factores: Creencia normativa, juicio acerca de lo que las personas importantes, referentes, para la persona opinan sobre la realización de una determinada conducta, y la Motivación para acomodarse a opinión de los referentes. El producto de las dos percepciones subjetivas indica el grado de influencia que la persona o personas de referencia tienen sobre la conducta en cuestión, a saber el juego de apuestas.

Otro factor que ha mostrado una reiterada evidencia en la explicación de conductas potencialmente adictivas es la accesibilidad. La mayor oferta de apuestas online a través de dispositivos móviles u ordenadores se ha relacionado con un aumento de la frecuencia de juego y predisposición al juego problemático (Clarke et al., 2006; Drakeford y Hudson, 2015; Engel, Bechtold, Kim y Mulvaney, 2013; Gainsbury, Wood, Russell, Hinga y Blaszczynski, 2012; Gainsbury et al., 2015; Lund, 2009; Orford, 2005; Rush, Veldhuizen y Adlaf, 2007; Storer, Abbott y Stubbs, 2009; Welte, Wierczorek, Barnes y Tidwell, 2006), aumentando especialmente los problemas de juego entre la población adolescente (King, Delfabbro y Griffiths, 2010; McBride y Derevensky, 2009). En cuanto al juego presencial, otros estudios también concluyen 
que existe una asociación entre la proximidad de casinos y salones de juego y la prevalencia de juego problemático (Shaffer, LaBrie y LaPlante, 2004).

A pesar de la alta prevalencia de juego de apuestas entre adolescentes españoles, son todavía escasos los estudios que describen los patrones de juego y no disponemos de suficiente evidencia sobre los factores que predisponen a su inicio y mantenimiento. El objetivo del presente trabajo es describir la conducta de juego de apuestas en una muestra de adolescentes y analizar su relación con la accesibilidad y la norma subjetiva. En consecuencia se proponen los siguientes objetivos específicos:

1) Analizar el tipo de juego según el sexo y la edad.

2) Describir la frecuencia de juego, según sexo y la edad.

3) Conocer la relación entre la accesibilidad a los juegos de apuestas y la frecuencia de juego.

4) Estudiar la asociación entre la norma subjetiva y la frecuencia con la que se juega.

Por lo anteriormente expuesto, las hipótesis planteadas en este estudio son:

Hipótesis 1. Se encontrará una relación directamente proporcional entre la edad y la frecuencia de juego.

Hipótesis 2. Una mayor accesibilidad a los juegos de apuestas, se relacionará con una mayor frecuencia de juego.

Hipótesis 3. La percepción de una norma subjetiva a favor del juego de apuestas se relacionará con un incremento en la frecuencia de juego.

\section{MÉTODO}

\section{Participantes}

Participaron 2.716 menores estudiantes de Educación Secundaria Obligatoria (ESO) y Bachillerato (BAT), pertenecientes a 15 centros distribuidos en 7 comarcas de la provincia de Alicante, manteniendo una ratio de dos centros por comarca. La media de edad fue de 15.12 años (DT = 1.03). La distribución por edades fue 13 años (6\%)
14 años (24\%) 15 años (29\%), 16 años (35\%) y 17 años (6\%). El $49.9 \%$ fueron chicas.

\section{Variables e instrumentos}

Frecuencia y Tipo de Juego. Cuestionario ad-hoc de 15 ítems adaptado de la encuesta europea ESPAD (ESPAD Group, 2016) que mide la frecuencia de juego indicando el número de veces que ha jugado en cada uno de los tres indicadores temporales: a) durante la vida; b) durante los últimos 12 meses; c) durante los últimos 30 días. Incluye 5 modalidades de juego: Apuestas deportivas on-line, Apuestas deportivas en salones y/o bares, Máquinas tragaperras en salones y/o bares, Póker o juegos de casino on-line y Ruletas en salones. La frecuencia de juego se categorizó en tres grupos: Nunca han jugado (67.8\%); Baja Frecuencia (18.1\%) definida por haber jugado a apuestas deportivas online o en salones o a máquinas tragaperras menos de cinco veces en la vida y menos de cuatro en los últimos 12 meses, y no haber jugado nunca a Póker o juegos de casino on-line ni a ruletas en salones; y Alta Frecuencia (14.1\%) incluye los que han jugado a apuestas deportivas online o en salones o a máquinas tragaperras cinco o más veces en la vida o cuatro o más en los últimos 12 meses, o han jugado alguna vez a Póker o juegos de casino on-line y a ruletas en salones.

Accesibilidad. Se midió con la subescala "accesibilidad" del cuestionario Early Detection Of Gambling Abuse Risk Among Adolescents (EDGAR, Lloret, Cabrera-Perona y Núñez, 2018). Evalúa la facilidad para acceder a juegos de apuestas. La escala la componen 6 ítems: 4 referentes a la accesibilidad al juego como concepto general, por ejemplo "Sabría a dónde dirigirme si quisiera apostar" y 2 relativos a juego presencial en salones y juego online. Las frases están enunciadas en positivo y en negativo, al objeto de evitar el llamado efecto de "aquiescencia autoadministrada". Cada ítem se responde en una escala de 5 alternativas de "muy en desacuerdo" a "muy de acuerdo" (a de Cronbach = .67).

Norma Subjetiva. Para evaluar el juicio sobre lo que las personas significativas opinan sobre la propia conducta de juego, se construyó un cuestionario siguiendo las recomendaciones propuestas por Ajzen (2002). Los referentes propuestos a los adolescentes fueron: padres, amigos/as, compañeros/as, pareja y profesores/as. Consta de dos dimensiones: a) percepción del grado de acuerdo de las personas más cercanas con el juego de apuestas, y b) valoración de la opinión de 
estas personas. Estas dos dimensiones se combinan de forma multiplicativa para obtener una puntuación única de la siguiente forma ( $a$ denota los ítems de la dimensión creencias y $b$ los de la dimensión valoración de la opinión):

$$
\text { Norma subjetiva }=\frac{\sum_{1}^{n} N S_{N}}{n . \text { items }}=\frac{\left(a_{1} \times b_{1}\right) / 5+\ldots+\left(a_{n} \times b_{n}\right) / 5}{n . \text { items }}
$$

Los ítems se responden mediante una escala Likert de 5 puntos (Muy desacuerdo Nada importante - Muy de acuerdo o Muy importante). La consistencia interna obtiene un a de Cronbach $=.73$.

\section{Procedimiento}

Se solicitó la aprobación de los centros para llevar a cabo la evaluación, así como, el consentimiento informado de los padres o tutores de los participantes y la autorización de la Autoridad competente en Educación y del Comité de Ética de la Universidad Miguel Hernández (UMH). Los sujetos participaron de forma voluntaria en la prueba tras haber sido informados de la finalidad del estudio.

La asignación de los centros por comarcas fue al azar. Una vez identificado cada centro, se solicitó la participación de la totalidad de alumnos de los cursos $3^{\circ}$ y $4^{\circ}$ de ESO y $1^{\circ}$ de BAT. El acceso a la muestra se realizó en las aulas de los centros educativos. La duración de las sesiones fue de 50 minutos aproximadamente, donde se cumplimentó la prueba de forma colectiva en las correspondientes aulas de los centros participantes, bajo supervisión del investigador y del tutor. El trabajo de campo se realizó durante los meses de septiembrenoviembre de 2016 .

\section{Análisis de datos}

Para los objetivos 1 y 2 los resultados se describen mediante frecuencia absoluta y porcentaje. Las diferencias entre sexos se determinaron con la prueba chi cuadrado $\left(X^{2}\right)$. Para los objetivos 3 y 4 se asume la normalidad de la distribución por el Teorema Central del Límite y se calcula ANOVA y MANOVA, y a continuación la prueba post-hoc de Tukey. Utilizamos la d de Cohen para cuantificar la magnitud de las diferencias. Los análisis se han realizado con el programa estadístico SPSS v24.0

\section{RESULTADOS}

En primer lugar, respecto a los objetivos 1 y 2, se compara la frecuencia de juego en los últimos 30 días en cada tipo de juego presencial $u$ on-line entre chicos y chicas y por edades. En términos generales, los chicos presentan prevalencias de juego significativamente mayores que las chicas. Tomando la totalidad de la muestra estudiada, el porcentaje de chicos que declara haber jugado en los últimos 30 días a cualquiera de las cinco modalidades es el $46.4 \%$, que duplica ampliamente el de las chicas (18.7\%). En cuanto a la prevalencia de juego según la edad, es a partir de los 16 años cuando se registra un notable aumento de la proporción de jóvenes de ambos sexos que declaran jugar. Sin embargo, teniendo en cuenta el tipo de juego, destaca el crecimiento del porcentaje de chicos que juega a apuestas deportivas en salones y/o bares, que de los 13 a los 16 años se duplica anualmente. Las máquinas tragaperras mantienen una presencia residual que aunque registra un leve aumento con la edad, no lo hace al mismo ritmo que las otras modalidades (Tabla 1).

Tabla 1. Adolescentes que han jugado en los últimos 30 días a diferentes tipos de juego según el sexo y edad

\begin{tabular}{|c|c|c|c|c|c|c|c|c|c|c|c|c|c|c|c|c|c|c|c|}
\hline & \multicolumn{5}{|c|}{$13-14$} & \multicolumn{5}{|c|}{15} & \multicolumn{4}{|c|}{16} & \multicolumn{5}{|c|}{$17-18$} \\
\hline & \multicolumn{2}{|c|}{$0^{x}$} & \multicolumn{2}{|l|}{ q } & \multirow[b]{2}{*}{$x^{2}$} & \multicolumn{2}{|c|}{$0^{x}$} & \multicolumn{2}{|l|}{ q } & \multirow[b]{2}{*}{$x^{2}$} & \multicolumn{2}{|l|}{$0^{x}$} & \multicolumn{2}{|c|}{ q } & \multicolumn{2}{|l|}{$0^{n}$} & \multicolumn{2}{|l|}{ q } & \multirow[b]{2}{*}{$x^{2}$} \\
\hline & $\%$ & $\mathrm{n}$ & $\%$ & $\mathrm{n}$ & & $\%$ & $n$ & $\%$ & $\mathrm{n}$ & & $\%$ & $n$ & $\%$ & $x^{2}$ & $\%$ & $\mathrm{n}$ & $\%$ & $\mathrm{n}$ & \\
\hline Apuestas deportivas on-line & 9.6 & 35 & 1.7 & 72 & $23.511^{* * *}$ & 7.9 & 35 & 3.0 & 12 & $9.322^{* *}$ & 9.6 & 38 & 1.8 & $825.627^{\star \star \star}$ & 12.8 & 153 & 3.1 & 4 & $8.032^{* \star}$ \\
\hline $\begin{array}{l}\text { Apuestas deportivas en salones y/o } \\
\text { bares }\end{array}$ & 6.6 & 24 & 2.4 & 10 & $7.930^{* *}$ & 12 & 53 & 3.6 & 14 & $20.203^{* \star *}$ & 21.1 & 83 & 2.6 & $1272.162^{\star \star \star}$ & 28.2 & 33 & 9.41 & 121 & $14.455^{* * *}$ \\
\hline $\begin{array}{l}\text { Máquinas tragaperras en salones y/o } \\
\text { bares }\end{array}$ & 3.0 & 11 & 1.2 & 5 & 3.097 & 5.0 & 22 & 2.0 & 8 & $5.229^{*}$ & 4.6 & 18 & 0.9 & $411.387^{* * *}$ & 5.1 & 63 & 3.1 & 4 & .627 \\
\hline Póker o juegos de casino on-line & 2.5 & 9 & 1.2 & 5 & 1.796 & 4.1 & 8 & 1.0 & 4 & $7.598^{* *}$ & 6.3 & 25 & 1.3 & $615.165^{* * *}$ & 11.1 & 135 & 5.5 & 7 & 2.596 \\
\hline Ruletas en salones & 0.6 & 2 & 0.7 & 3 & .100 & 3.2 & 14 & 1.5 & 6 & 2.413 & 4.6 & 18 & 0.9 & $411.387^{* * *}$ & 15.4 & 183 & 3.9 & 5 & $9.468^{*}$ \\
\hline
\end{tabular}

Nota: ${ }^{* * *} p \leq .001 ;{ }^{* *} p \leq .01 ;{ }^{*} p \leq .05$ 
En el global de juego de apuesta, la Figura 1 muestra una comparativa por sexo y edad. Pueden observarse diferencias significativas por sexo en todas las edades, donde los chicos juegan en mayor proporción, tanto en el grupo de baja como en el de alta frecuencia. En los grupos de alta frecuencia, respecto a los chicos son los 16 años donde se produce un amplio ascenso, mientras que en el de las chicas, éste se produce a los 17 años.

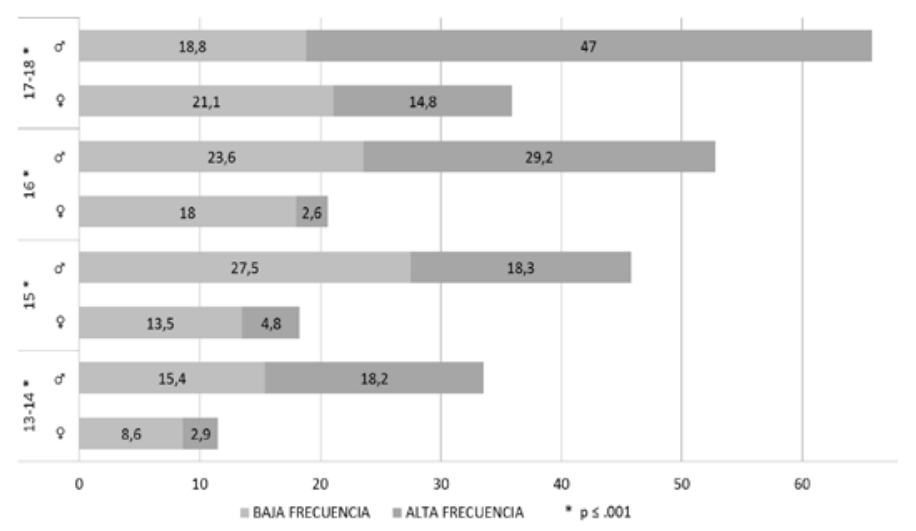

En el tercer objetivo se analiza la relación entre la accesibilidad a los juegos de apuestas y la frecuencia de juego. La prueba de ANOVA determina que existen diferencias entre los tres grupos (F: 68,384, p=.000) Para conocer las diferencias en la escala de accesibilidad entre cada uno de los tres grupos de frecuencia tomados de dos en dos, se realiza un contraste de medias (Tabla 2). Todas las diferencias son significativas $(p=.000)$, lo que sugiere la potencia de la variable accesibilidad para explicar la conducta de juego. Como era de esperar, la mayor diferencia se observa al comparar a los que nunca han jugado con los que lo hacen con mayor frecuencia. La magnitud del efecto $(d=.65)$ indica que la percepción de la facilidad de acceso al juego del $77 \%$ de los adolescentes que nunca han jugado es inferior a la media del grupo de jugadores más frecuentes.

Figura 1. Porcentaje de frecuencia de juego (alta/baja) según sexo y edad

Tabla 2. Prueba Post-hoc HSD Tukey para la Percepción de la accesibilidad según la frecuencia de juego

\begin{tabular}{|c|c|c|c|c|c|c|c|c|}
\hline \multicolumn{9}{|l|}{ Frecuencia Juego } \\
\hline \multirow[b]{2}{*}{ (l) } & \multirow[b]{2}{*}{ (J) } & \multirow[b]{2}{*}{$\mathrm{M}(\mathrm{I}) \mathrm{DT}$} & \multirow[b]{2}{*}{$M(J) D T$} & \multirow{2}{*}{$\begin{array}{l}\text { Diferencia medias } \\
\qquad(I-J)\end{array}$} & \multirow[b]{2}{*}{ Desv. Error } & \multicolumn{2}{|c|}{ Intervalo confianza al 95\% } & \multirow[b]{2}{*}{$d$} \\
\hline & & & & & & Límite inf. & Límite sup & \\
\hline Nunca(N=1695) & Baja(N=454) & $2.392(.698)$ & $2.588(.662)$ & $-.19565^{* \star}$ & .03661 & -.2815 & -.1098 & .28 \\
\hline Nunca(N=1695) & Alta(N=353) & $2.392(.698)$ & $2.848(.703)$ & $-.45515^{* *}$ & .04053 & -.5502 & -.3601 & .65 \\
\hline Baja $(N=454)$ & Alta(N=353) & $2.588(.662)$ & $2.848(.703)$ & $-.25950^{* *}$ & .04916 & -.3748 & -.1442 & .38 \\
\hline
\end{tabular}

Nota: ${ }^{* \star} \mathrm{p} \leq .001 ; \mathrm{d}: \mathrm{d}$ de Cohen; DT: Desviación típica; N: Tamaño muestral; M: Media.

Tabla 3. Prueba Post-hoc HSD Tukey para la Percepción de la Norma Subjetiva según la frecuencia de juego

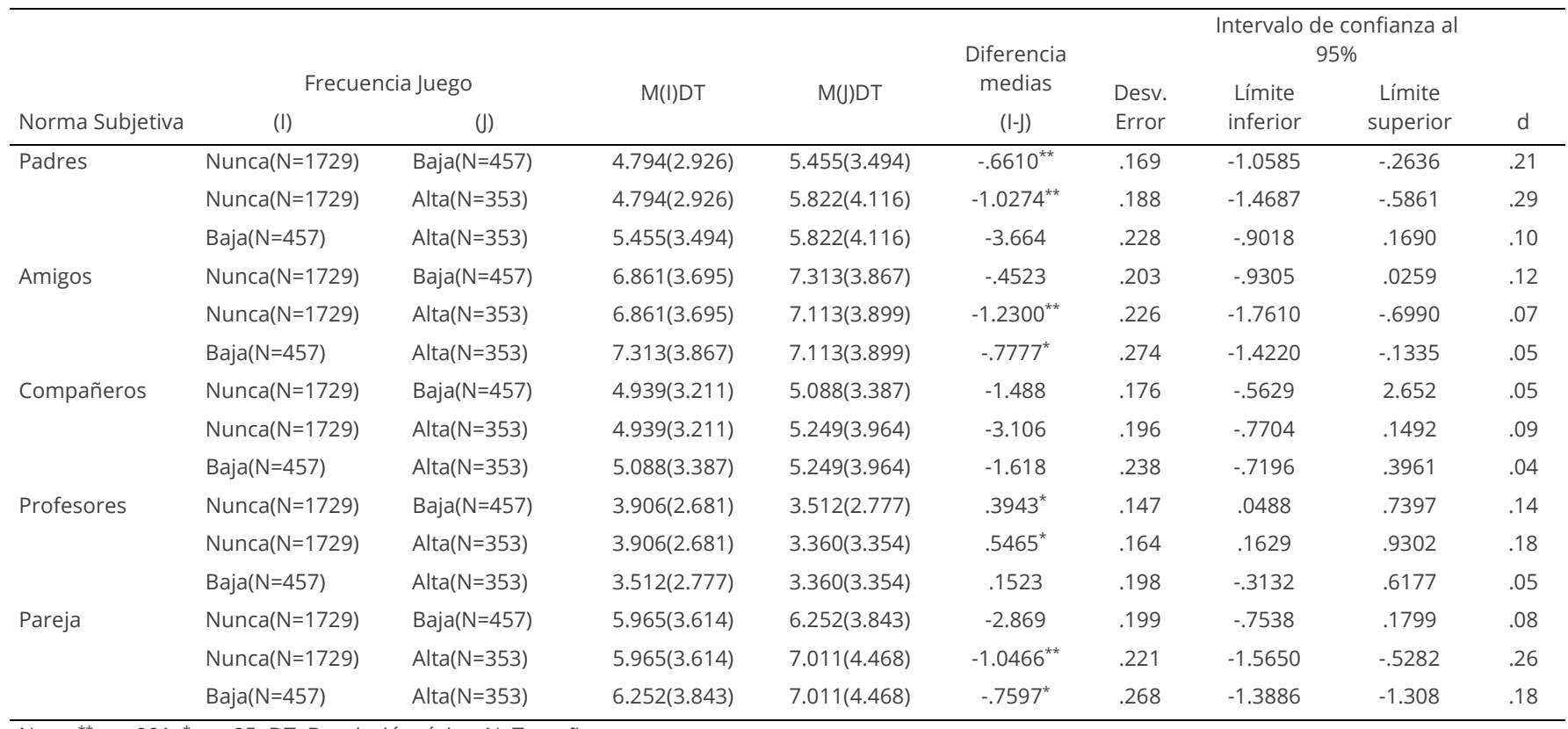

Nota: ${ }^{* *} \mathrm{p} \leq .001 ;{ }^{*} \mathrm{p} \leq .05 ; \mathrm{DT}$ : Desviación típica; N: Tamaño 
Para analizar la relación entre la frecuencia de juego y la norma subjetiva se identificaron cinco grupos de personas significativas: padres, amigos, compañeros, pareja y profesores. En la Tabla 3 se muestra el resultado de la prueba MANOVA y su correspondiente post-hoc de Tukey. Los análisis comparan las puntuaciones de cada uno de los tres grupos de frecuencia de juego. Los referentes de mayor influencia fueron los padres/madres, los amigos y la pareja, aunque las magnitudes de las diferencias fueron bajas en el mejor de los casos. Los colectivos de profesores y compañeros apenas mostraron diferencias significativas y cuando lo hicieron, la magnitud de estas no alcanza el criterio mínimo de .20 para ser consideradas.

\section{DISCUSIÓN}

El objetivo del presente estudio era describir la conducta de juego de apuestas en una amplia muestra de adolescentes y analizar su relación con la accesibilidad y la norma subjetiva. El análisis de la conducta de juego se centró en la frecuencia según el tipo de juego.

En cuanto a tipos de juego destacan la baja prevalencia de las máquinas tragaperras, a pesar de que tradicionalmente han sido uno de los métodos de apuestas favorito entre jugadores. Esta modalidad de apuesta registra una baja penetración entre los adolescentes, y su uso no aumenta con la edad como ocurre con otros tipos de juego. Se observa, por tanto una mayor preferencia en los adolescentes por apuestas que requieran algún tipo de estrategia de juego (Wilber y Potenza, 2006). La mayor preferencia por realizar apuestas deportivas (online y presenciales) entre los jóvenes participantes en el estudio es coherente con los resultados obtenidos por otros autores (Chóliz y Lamas, 2017; Lupu y Todirita, 2013; Sarabia, Estévez y Herrero, 2014; Ricijas, Hundric y Huic, 2016).

En este caso, los datos confirmarían nuestra hipótesis 1 para todas las edades en apuestas deportivas (presenciales y online), para 15-16 años respecto a juegos de casino online y 16-18 años en cuanto a ruleta en casinos. Por sexos, los chicos presentaron frecuencias superiores en todos los tipos de juego, siendo significativamente mayores en las apuestas deportivas en las cuatro edades estudiadas. Estos datos son coherentes con los hallados en investigaciones con muestras similares (Chóliz y Lamas, 2017; Rahman, et al., 2012; Mateu, 2016).

En términos generales, en ambos sexos la frecuencia de juego aumenta conforme los adolescentes crecen (García, 2015; Kong, et al., 2013) y de forma especial a los 16 años. Estos datos coinciden con los reportados por otros autores, donde se indica que entre los 15 y los 17 años hay un notable incremento de la frecuencia de juego, siendo mayor en chicos (Delfabbro, Lahn y Grabosky, 2005).

Respecto a la accesibilidad o facilidad con que los jóvenes perciben el acceso a las casas de apuestas, se constata que se relaciona con una alta frecuencia en el juego de apuestas. Así, nuestros datos confirmarían la hipótesis 2. La accesibilidad es un factor de riesgo de primera magnitud, que explica en gran parte el incremento constante en el número de menores que apuestan y de jugadores patológicos (Clarke et al., 2006; Drakeford y Hudson, 2015; Engel et al., 2013; Gainsbury et al. 2012; Gainsbury et al., 2015; Lund, 2009; Orford, 2005; Rush et al., 2007; Storer et al., 2009; Welte et al., 2006).

Los resultados sostienen también nuestra hipótesis 3, ya que la percepción de la norma subjetiva en los padres, el grupo de iguales o la pareja se relaciona proporcionalmente con la frecuencia de juego, en línea con otros estudios en los que se muestra que la norma social es un buen predictor de la frecuencia de juego (Martin, et al. 2011; Moore y Ohtsuka, 1999; Secades y Villa, 1998; Wu y Tang, 2012). La amplia difusión del juego de apuestas a través de los medios de comunicación, estaría en la base de la normalización del juego de apuestas que explicaría por qué los adolescentes creen que tanto sus familiares como amigos aprueban que se pueda jugar.

Así, en nuestra muestra se dibujaría un perfil de mayor frecuencia de juego cuando se percibe el juego de apuestas como una conducta normalizada, de gran oferta de modalidades, accesible y hacia los que los referentes próximos del menor muestran una actitud favorable. Esta frecuencia de juego aumentaría con la edad y en mayor medida en los chicos.

Los resultados deben interpretarse teniendo en cuenta las limitaciones propias de los estudios transversales autoinformados. La toma de datos en un único momento solo permite sugerir relaciones entre 
variables. Habría sido deseable incluir otros colectivos con el fin de evaluar las actitudes hacia el juego, y contrastar la percepción de las creencias en la medida de la norma subjetiva. Por último, es necesario advertir que la selección muestral por conveniencia supone un obstáculo a la generalización de los resultados. Habría sido deseable incluir alumnado de Ciclos Formativos (FP) así como menores no incluidos en el sistema educativo regular.

Estas limitaciones sugieren futuras líneas de investigación que integren diferentes colectivos, como padres/madres e hijo/as, y permitan contrastar las opiniones de unos sobre otros. Así mismo, para determinar relaciones predictivas sería necesario un diseño longitudinal en el que se incluya un mayor número de variables.

En cuanto a la aplicabilidad de los resultados, atendiendo a la relación entre edad y juego, los datos sugieren que las intervenciones preventivas de ámbito universal deben dirigirse preferentemente a menores de 15 años. Nuestros datos y los de investigaciones similares (García, 2015; Kong, et al., 2013) detectan un importante aumento del juego a partir de esta edad, lo que significa una consolidación de las actitudes hacia el juego de apuestas. Una mayor experiencia de apuestas puede ayudar a fijar creencias como son las expectativas de éxito y reducir la percepción del riesgo. La sensibilización es una estrategia fundamental en los programas de prevención, máxime cuando se trata de prevención universal dirigida a edades en las que aún no ha habido experiencia de juego. Desde una perspectiva de sexo, el evidente dimorfismo sexual de la conducta de juego debe ser tenido en cuenta en el diseño e implementación de acciones preventivas.

Por último, los datos acreditan la alta percepción que tienen los adolescentes de la facilidad de acceso y su relación directamente proporcional con frecuencia de juego. Así, esta percepción de accesibilidad y acuerdo con la norma subjetiva podría estar relacionada con una normalización de la conducta de apuestas, en la que una mayor concesión de licencias de apertura de salones, un mayor número de webs y casas de apuestas online y la presión mediática a la que se ven sometidos los menores pudiera estar actuando como variables mediadoras (King, Delafabro y Griffith, 2010). Esta evidencia debe ser entendida como una llamada de atención a reguladores y administraciones públicas para que refuercen sus esfuerzos para reducir la presencia y disponibilidad de los juegos de apuestas. La puesta en marcha de medidas de control de la oferta ha sido revindicada por diferentes autores que proponen recomendaciones concretas en este sentido.

\section{REFERENCIAS}

Ajzen, I. (1987). Attitudes, traits and actions: Dispositional Prediction of Behavior in Personality and Social Psychology. Advances in Experimental Social Psychology, 20, 1-63. DOI https://doi.org/10.1016/S0065-2601(08)60411-6

Ajzen, I. (2002). Constructing a TPB questionnaire: Conceptual and methodological considerations. Recuperado de la Red en: https://pdfs.semanticscholar.org/0574/b20bd58130dd5a961f1a2d b10fd1fcbae95d.pdf

Becoña, E., Míguez, M.C. \& Vázquez, F.L. (2001). El juego problema en los niños escolarizados de la enseñanza secundaria de 14 y más años de Galicia. Psicothema. 13, (4), 559-564.

Calado, F., Alexandre, J. \& Griffiths, M.D. (2017). Prevalence of Adolescent Problem Gambling: A Systematic Review of Recent Research. Journal of Gambling Studies, 22(2), 397-424.

Chóliz, M. \& Lamas, J. (2017) ¡Hagan juego, menores! Frecuencia de juego en menores de edad y su relación con indicadores de adicción al juego. Revista Española de Drogodependencia, 42 (1), 34-47.

Clarke, D. (2006). Impulsivity as a mediator in the relationship between depression and problem gambling. Personality and Individual Differences, 40 (1), 5-15.

Cox, B.J., Enns, M. W. \& Michaud, V. (2004). Comparisons between the south oaks gambling screen and a DSM-IV-based interview in a community survey of problem gambling. Canadian Journal of Psychiatry, 49 (4), 258-64.

Delfabbro, P., Lahn, J. \& Grabosky, P. (2005). Adolescent Gambling in the ACT. Centre for Gambling Research: The Australian National University. Camberra: Australia.

Dirección General de Ordenación del Juego (2015a). Informe Anual 2015. Ministerio de Hacienda y Administraciones Públicas. Madrid, España.

Dirección General de Ordenación del Juego (2015b). Estudio sobre prevalencia, comportamiento y características de los usuarios de juego de azar en España. Ministerio de Hacienda y Administraciones Públicas. Madrid, España.

Dirección General de Ordenación del Juego (2017). Informe Trimestral. Mercado del juego online en España (Trimestre 3). Ministerio de Hacienda y Función Pública. Madrid, España.

Dixon, R.W., Youssef, G.J., Hasking, P., Yucel, M., Jackson, A.C., \& Dowling, N. A. (2016). The relationship between gambling attitudes, involvement, and problems in adolescence: examining the moderating role of coping strategies and parenting styles. Addictive Behaviors, 58, 42-46. doi: 10.1016/j.addbeh.2016.02.011 
Donati, M. A., Chiesi, F. \& Primi, C. (2013). A model to explain atrisk/problem gambling among male and female adolescents: Gender similiarities and differences. Journal of Adolescence, 36, DOI:129-137.10.1016/j.adolescence.2012.10.001

Drakeford, B.P. \& Hudson, M. (2015). Mobile Gambling: Implications of Accessibility. Journal of Research Studies in Business \& Management, $1(1), 3-28$.

Dussault, F., Brendgen, M., Vitaro, F., Wanner, B. \& Tremblay, R. E. (2011). Longitudinal links between impulsivity, gambling problems and depressive symptoms: a transactional model from adolescence to early adulthood. Journal of Child Psychology and Psychiatry, 52, 130138

Engel, R., Bechtold, J., Kim, Y. \& Mulvaney, E. (2013). Beating the Odds: Preparing Graduates to Address Gambling-Related Problems. Journal of Social Work Education, 48 (2), 321-335.

ESPAD Group (2016). ESPAD Report 2015 Results from the European School Survey Project on Alcohol and Other Drugs. Lisbon, Portugal: European Monitoring Centre on Drugs and Drugs Addiction.

Gainsbury, S., Wood, R., Russell, A., Hinga, N., \& Blaszczynski, A. (2012). A digital revolution: Comparison of demographic profiles, attitudes and gambling behavior of Internet and non-Internet gamblers. Computers in Human Behavior, 28 (4), 1388-1398. DOI: https://doi.org/10.1016/j.chb.2012.02.024

Gainsbury, S.M., Russell, A., Hing, N., Wood, R., Lubman, D. \& Blaszczynski, A. (2015). How the Internet is Changing Gambling: Findings from an Australian Prevalence Survey. Journal of Gambling Studies, 31 (1), 115

García, P., Buil, P. y Solé, M.J. (2015). Consumos de riesgo: menores y juegos de azar online. El problema del "juego responsible". Política y Sociedad, 53(2), 551-575.

Gill, T., Grande, E.D., \& Taylor, A.W. (2006). Factors associated with gamblers: a population-based cross-sectional study of south Australian adults. Journal of Gambling Studies, 22(2), 143-164.

González-Ros, A., Fernández-Hermida, J. R., Weidberg, S., Martínez-Loredo, V. \& Secades-Villa, R. (2016). Prevalence of Problem gambling among adolescents: a comparison across models of Access, gambling activities, and levels of severity. Journal of Gambling Studies, 33(2), 371-382 Doi: 10.1007/s10899-016-9652-4

Kessler, R.C. Hwang, I., LaBrie, R., Petukhova, M., Sampson, N.A., Winters, K.C. \& Shaffer, H.J. (2008). DSM-IV pathological gambling in the National Comorbidity Survey Replication. Psychological Medicine. 38(9), 1351-1360

King D., Delfabbro P. \& Griffiths M. (2010). The convergence of gambling and digital media: Implications for gambling in young people. Journal of Gambling Studies, 26(2), 175-187.

Kong, G., Tsai, J., Pilver, C.E., Sim, H., Hoff, R.A., Cavallo, D., et al. (2013). Differences in gambling problem severity and gambling and health/functioning characteristics among Asian-American and Caucasian high-school students. Psychiatry Res. 210(3), 1-16.

Lloret, D. Cabrera-Perona, V. \& Núñez, R. (2018). Early Detection of Gambling Abuse Risk among Adolescents. Validation of Edgar_A Scale. International Gambling Studies. (Inédito).
Lloret, D. Cabrera-Perona, V., Castaños, A., Segura, J.V., Antón, M.A. \& Caselles, P. (2018). El juego de apuestas en adolescentes de la provincia de Alicante II. Estudio longitudinal de los hábitos de juegos de apuestas y los predictores psicosociales. Diputación de Alicante.

Lund, I. (2009).Gambling Behaviour and the Prevalence of Gambling Problems in Adult EGM Gamblers when EGMs are Banned. A Natural Experiment. Journal of Gambling Studies, 25(2), 215-225.

Lupu, V., \& Todirita, I.R. (2013). Updates of the prevalence of problem gambling in Romanian teenagers. Journal of Gambling Studies, 29 (1), 29-36.

Martin, R.J., Nelson, S., Usdan, S., \& Turner, L. (2011). Predicting college student gambling frequency using the theory of planned behavior: Does the theory work differently for disordered and nondisordered gamblers? Analysis of Gambling Behaviour, 5, 45-58.

Mateu, J. (2016). Análisis sobre el aumento de las apuestas deportivas en adolescentes estudiantes y las conductas de riesgo asociadas. Actividad física y deporte: Ciencia y Profesión. 24, 41-52.

McBride, J. \& Derevensky, J. (2009). Internet Gambling Behavior in a Sample of Online Gamblers. International Journal of Mental Health \& Addiction, 7 (1), 149-167. 19. DOI: 10.1007/s11469-008-9169-x.

Míguez, M.C. \& Becoña, E. (2015). Do cigarette smoking and alcoho consumption associate with cannabis use and problem gambling among Spanish adolescents? Adicciones, 27(1), 8-16.

Ministerio de Hacienda y Administraciones Públicas (2011). Ley 13/2011 de Regulación del Juego. Recuperado de la Red en: https://www.boe.es/buscar/pdf/2011/BOE-A-2011-9280consolidado.pdf

Moore, S. \& Ohtsuka, K. (1999). The prediction of gambling behaviour and problem gambling from attitudes and perceived norms. Social Behavior and Personality, 27, (12) 455-466. DOI: https://doi.org/10.2224/sbp.1999.27.5.455

Molinaro, S., Canale, N., Vieno, A., Lenzi, M., Siciliano, V., Gori, M., et al. (2014). Country and individual-level determinants of probable problematic gambling in adolescence: A multi-level cross-national comparison. Addiction. 109 (12), 2089-2097.

Orford, J. (2005). "Complicity on the river bank: The search for truth about problem gambling: Reply to the commentaries". Addiction, 100 (9), 1235-1239. https://doi.org/10.1111/j.1360-0443.2005.01219.x

Potenza, M.N., Wareham, J.D., Steinberg, M.A., Rugle, L., Vavallo, D.A., Krishnan-Sarin, S. \& Desai, R.A. (2011). Correlates of at-risk/problem internet gambling in adolescents. Journal of the American Academy of Child and Adolescence Psychiatry. 50 (2), 150-159.

Rahman, A.S. et al. (2012). The relationship between age of gambling onset and adolescent problematic gambling severity. Journal of Psychiatric Research, 46, (5) 675-683. DOI: https://doi.org/10.1016/j.jpsychires.2012.02.007

Ricijas, N. Hundric, D.D. \& Huic, A. (2016). Predictors of adverse gambling related consequences among adolescent boys. Children and Youth Services Review, 67, 168-176. https://doi.org/10.1016/j.childyouth.2016.06.008

Rush, B., Veldhuizen, S. \& Adlaf, E. (2007). Mapping the prevalence of problem gambling and its association with treatment accessibility 
and proximity to gambling venues. Journal of Gambling Issues, 20, 193-213. DOI: http://dx.doi.org/10.4309/jgi.2007.20.6

Sarabia, I., Estévez, A. \& Herrero, D. (2014). Situación actual del juego con dinero en jóvenes y adolescentes. Revista Española de Drogodependencias, 39 (3), 57-68.

Shaffer, H.J., LaBrie, R.A. \& LaPlante, D. (2004). Laying the Foundation for Quantifying Regional Exposure to Social Phenomena: Considering the Case of Legalized Gambling as a Public Health Toxin. Psychology of Addictive Behaviors, 18 (1), 40-48 DOI: 10.1037/0893-164X.18.1.40

Storer, J., Abbott, M. \& Stubbs, J. (2009). Access or adaptation? A metaanalysis of survey of problem gambling prevalence in Australia and New Zealand with respect to concentration of electronic gaming machines. International Gambling Studies, 9 (3), 225-244.

Vitaro, F., Arseneault, L. \& Tremblay, R. E.(1999). Impulsivity predicts problem gambling in low SES adolescent males. Addiction, 94 (4), 565-575. https://doi.org/10.1046/j.1360-0443.1999.94456511.x

Welte, J., Wierczorek, F., Barnes, G. \& Tidwell, M. (2006). Multiple Risk Factors for Frequent and Problem Gambling: Individual, Social and Ecological. Journal of Applied Social Psychology, 36(6), 1548-1568. https://doi.org/10.1111/j.0021-9029.2006.00071.x

Wiebe, J.M., \& Cox, B.J. (2005). Problem and probable pathological gambling among older adults assessed by the SOGS-R. Journal of Gambling Studies, 21(2), 205-221. Recuperado de: http://dx.doi.org/10.1007/s10899-005-3032-9.

Wilber, M.K. \& Potenza, M.N. (2006). Adolescent Gambling. Research and Clinical Implications. Psychiatry (Edgmont). 3 (10), 40-48.

Wu, A.M., \& Tang, C. S. (2012). Problem gambling of Chinese college students: Application of the theory of planned behaviour. Journal of Gambling Studies, 28 (2), 315-324. 